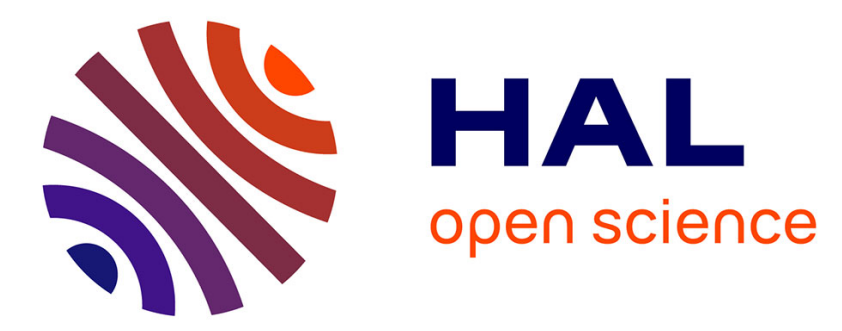

\title{
In vivo ultrasound characterization of red blood cell aggregation using the structure factor size and attenuation estimator
}

Emilie Franceschini, François T. H. Yu, François Destrempes, Guy Cloutier

\section{- To cite this version:}

Emilie Franceschini, François T. H. Yu, François Destrempes, Guy Cloutier. In vivo ultrasound characterization of red blood cell aggregation using the structure factor size and attenuation estimator. Proceedings of the 2009 IEEE International Ultrasonics Symposium (Italie Rome), Sep 2009, Italy. pp.301-304. hal-00443783

\section{HAL Id: hal-00443783 \\ https://hal.science/hal-00443783}

Submitted on 4 Jan 2010

HAL is a multi-disciplinary open access archive for the deposit and dissemination of scientific research documents, whether they are published or not. The documents may come from teaching and research institutions in France or abroad, or from public or private research centers.
L'archive ouverte pluridisciplinaire HAL, est destinée au dépôt et à la diffusion de documents scientifiques de niveau recherche, publiés ou non, émanant des établissements d'enseignement et de recherche français ou étrangers, des laboratoires publics ou privés. 


\title{
In vivo ultrasound characterization of red blood cell aggregation using the Structure Factor Size and Attenuation Estimator
}

\author{
Emilie Franceschini ${ }^{1}$, François T. H. Yu ${ }^{2}$, François Destrempes ${ }^{2}$ and Guy Cloutier ${ }^{2}$ \\ ${ }^{1}$ Laboratory of Mechanics and Acoustics, LMA - CNRS UPR 7051, Marseille, France \\ ${ }^{2}$ Laboratory of Biorheology and Medical Ultrasonics, University of Montreal Hospital \\ Research Center (CRCHUM), \\ Montreal, Canada. \\ Email: franceschini@1ma.cnrs-mrs.fr
}

\begin{abstract}
The ultrasound characterization of red blood cell (RBC) aggregation is an attractive tool to determine rheological blood flow disorders in vivo and in situ. The backscattered signals from blood can be spectrally analyzed to deduce the size and spatial distribution of RBC aggregates. One difficulty to apply this analysis in vivo is due to the frequency-dependent attenuation caused by intervening tissue layers that distorts the spectral content of backscattered echoes from blood microstructures. An optimization method was recently proposed to simultaneously estimate tissue attenuation and blood structure properties, and was termed the Structure Factor Size and Attenuation Estimator (SFSAE). With in vitro experiments, the method gave satisfactory estimates with relative errors below $25 \%$ for attenuations between 0.115 and $0.411 \mathrm{~dB} / \mathrm{MHz}$ and $k R<2.08$ ( $k$ being the wave number and $R$ the aggregate radius). The current work presents in vivo results to demonstrate the feasibility of the method.

Measurements were performed on an arm's vein of a normal subject using an ultrasound scanner equipped with a $25 \mathrm{MHz}$ center frequency probe. The probe was positioned in longitudinal view to examine three blood flow conditions: normal blood flow, stop flow with a proximal and distal constrictions of the ROI, and finally complex flow in the vinicity of two closed venous valves. For each case, the aggregate diameter $D$, expressed in number of RBCs, the packing factor $W$ and the total attenuation $\alpha_{0}$ were estimated by using the SFSAE, i.e. by comparing the spectrum of the backscattered radio-frequency echoes with newly developed models of RBC aggregate scattering.

Quantitative ultrasound parametric images of $D, W$ and $\alpha_{0}$ estimates were constructed. For the two structural parameters $D$ and $W$, statistically significant differences were observed between normal and
\end{abstract}


stopped flow conditions, as well as between blood stagnation and circulation zones in the case of the two closed venous valves. To conclude, this work shows the SFSAE ability to estimate blood backscattering properties in vivo and in situ, and opens the way to parametric imaging for clinical studies in abnormal blood conditions.

Keywords: ultrasound backscatter, structure factor, attenuation, in vivo, blood aggregation

\section{INTRODUCTION}

Ultrasonic (US) backscattered echoes from blood contain frequency-dependent information that can be used to obtain quantitative parameters reflecting the aggregation state of red blood cells (RBCs). Such quantitative parameters would be of great interest to elucidate the impact of RBC aggregation on several circulatory diseases (such as deep venous thrombosis and diabetes mellitus) in vivo and in situ. Two parameters that are estimated using the power spectrum of the echoes are the mean aggregate diameter and the packing factor [1]. Unfortunately, determining these two parameters in in vivo conditions is difficult because the spectral content of backscattered echoes is also affected by attenuation caused by intervening tissue layers between the probe and the blood flow. More generally, ultrasound scatterer size estimation techniques for tissue characterization (such as liver, kidney, prostate or breast) are facing similar challenges. The best clinical successes were obtained with tumors of the eye when the attenuation could be neglected [2]. Recently, Bigelow and co-workers [3] have introduced a new algorithm that has the advantage to estimate the characteristic size of the tissue microstructure and the total attenuation along the propagation path simultaneously. In our previous work, a similar strategy was adapted for estimation of RBC scatterer sizes [4]. Three parameters (the packing factor, mean fractal aggregate diameter and total attenuation) were determined simultaneously by using a minimization method that fits

the spectrum of the backscattered rf echoes from the region of interest (ROI) to an estimated spectrum by an appropriate model, termed the Structure Factor Size and Attenuation Estimator (SFSAE). The SFSAE was experimentally validated on porcine blood sheared in a Couette flow device with intervening tissue mimicking phantoms.

The goal of this paper was to assess the SFSAE ability to evaluate blood structural properties and total attenuation under in vivo conditions. Ultrasound measurements were realized in the range of 10 to 30 $\mathrm{MHz}$ on an arm's vein of a normal subject. Three cases were examined: normal blood flow, stop flow obtained from a proximal and distal constrictions of the vein, and finally complex flow in the vinicity of two closed venous valves. For each case, the blood structure properties and the total attenuation were 
estimated by the SFSAE and were compared with the expected aggregate size depending on the flow conditions.

\section{BACKGROUND: THE STRUCTURE FACTOR SIZE AND ATTENUATION ESTIMATOR}

US scattering from blood is mainly caused by the RBCs. These RBCs cannot be treated as independent scatterers since their concentration is very important (i.e. 35 to $45 \%$ ) under normal physiological conditions. The theoretical model of ultrasound backscattering by blood that we developped [1] is based on the particle approach [5], [6], which consists of summing contributions from individual RBCs and modeling the RBC interaction by a particle pair-correlation function. Assuming that all the RBCs in the insonified blood are identical and using the Born approximation (weak scattering), the theoretical backscatter coefficient from blood is given by [4]:

$$
B S C_{\text {theor }}(k)=m \sigma_{b}(k) S(k) A(k)
$$

where $k$ is the wave vector, $m$ is the number density of RBCs in blood estimated by assuming an hematocrit $H$ of $40 \%\left(m=H / V_{s}\right.$, where $V_{s}$ is the volume of a RBC), $\sigma_{b}$ is the backscattering cross section of a single RBC, $S$ is the structure factor describing the spatial organization of RBCs, and $A$ is the frequency-dependent attenuation function. The structure factor $S$ is by definition the Fourier transform of the pair-correlation function [6] $g$ and can be approximated by its second-order Taylor expansion [1] in $k$ as

$$
S(k)=1+m \int(g(r)-1) e^{-2 j k r} d r \approx W-\frac{12}{5}(k R)^{2} .
$$

$W$ is the low-frequency limit of the structure factor $\left(\left.S(k)\right|_{k \rightarrow 0}\right)$ called the packing factor [6], [7]. $R$ is the radius of $3 \mathrm{D} \mathrm{RBC}$ aggregates assumed to be isotropic. We introduce $D=R / a$ as the isotropic diameter of an aggregate (expressed in number of RBCs) with $a$ the radius of one RBC sphere-shaped model of volume $V_{s}$. As a first approximation, we assume that the attenuation increases linearly with the frequency: $\alpha(f)=\alpha_{0} f$. The attenuation function $A$ is thus given by:

$$
A(k)=e^{-4 \alpha_{0} k \frac{c}{2 \pi}}=e^{-4 \alpha_{0} f},
$$

where $c$ is the mean speed of sound in the intervening tissue layers, $f$ is the frequency and $\alpha_{0}$ is the

attenuation coefficient (in $\mathrm{dB} / \mathrm{MHz}$ ) defined by: $\alpha_{0}=\sum_{i} \alpha_{i} e_{i}$, where $\alpha_{i}$ and $e_{i}$ are respectively the intervening tissue layer attenuations (in $\mathrm{dB} / \mathrm{cm} / \mathrm{MHz}$ ) and thicknesses. 
The measured backscatter coefficient reported in this study was computed as

$$
B S C_{\text {meas }}(k)=B S C_{\text {ref }}(k) \frac{\overline{P_{\text {meas }}(k)}}{\overline{P_{\text {ref }}(k)}} .
$$

In Eq. (4), the mean backscattered power spectrum $\overline{P_{\text {meas }}}$ was obtained by averaging the power spectra of 20 backscattered echoes from blood. The mean power spectrum $\overline{P_{r e f}}$ was obtained from a reference sample of non-aggregated RBCs at a low hematocrit of $6 \%$ (i.e. Rayleigh scatterers) [8]. In this case, 20 echoes were also averaged. The backscatter coefficient of this reference sample $B S C_{r e f}$ was estimated by using the expression of the Perkus-Yevick packing factor for spheres $W_{P Y s}$ [6] [7] that is a function of the hematocrit $H$. This reference sample was used to compensate the backscattered power spectrum $\overline{P_{\text {meas }}}$ for the electromechanical system response, and the depth-dependent diffraction and focusing effects of the US beam.

The packing factor $W$, aggregate diameter $D$ and total attenuation along the propagation path $\alpha_{0}$ were determined by matching the measured $B S C_{\text {meas }}$ given by Eq. (4) with the theoretical $B S C_{\text {theor }}$ given by Eq. (1). For this purpose, we searched values of $\left(W, D^{2}, \alpha_{0}\right) \in(0, \infty) \times(0, \infty) \times[0,1], W$ and $D$ being variables without dimension and $\alpha_{0}$ expressed in $\mathrm{dB} / \mathrm{MHz}$, minimizing the cost function which synthesizes all of the wavenumbers $k_{i}(i=1 \cdots N)$ within the $-20 \mathrm{~dB}$ bandwidth of $\overline{P_{\text {meas }}}$

$$
F\left(W, D^{2}, \alpha_{0}\right)=\sum_{i}\left\|B S C_{\text {meas }}\left(k_{i}\right)-B S C_{\text {theor }}\left(k_{i}\right)\right\|^{2} .
$$

To insure that the global minimum of the optimization strategy is found, we employed an exhaustive search on the value of $\alpha_{0}$ combined with an analytical solution for the two blood structure parameters given for a fixed value of the attenuation [9]. If the solution $\left(W, D^{2}, \alpha_{0}\right)$ of the optimization problem was such that $W$ or $D^{2}$ was on the inferior boundary of the domain (i.e. $W$ or $D^{2}$ are equal to zero), the solution was rejected since such values of $W$ or $D^{2}$ are unrealistic [9].

\section{EXPERIMENTAL SET-UP}

US measurements were performed on an arm's vein of a normal subject. An US scanner (Vevo 660, Visualsonics, Toronto, Canada) equipped with the RMV 710 probe was used in B-mode. This probe consists of an oscillating single-element focused circular transducer having a center frequency of 25 $\mathrm{MHz}$, a diameter of $7.1 \mathrm{~mm}$ and a focal depth of $15 \mathrm{~mm}$. We acquired RF data from this scanner at a sampling frequency of $250 \mathrm{MHz}$ with 8 bits resolution (Gagescope, model 8500CS, Montreal, Canada). The probe was positioned parallel to the vein of interest to provide a longitudinal section view of the 
vein, and its transducer focal point was placed at the middle of the vein. In the same ROI, two blood flow conditions were successively examined: normal blood flow (Fig. 1 a) and stopped flow with a proximal and distal constrictions of the vein (Fig. 1 b). In another ROI, two momentarily closed venous valves were imaged giving rise to a complex blood flow (Fig. $1 \mathrm{c}$ ). In this peculiar case, regions were examined upstream and downstream from the two venous valves.

For each blood flow, 20 B-mode images were constructed for 4 s. Each image contained 384 vertical lines. For the normal and stop flows, 200 vertical lines were taken in the center of the B-mode images and echoes were selected with a rectangular window of length $0.4 \mathrm{~mm}$ at 85 depths every $0.031 \mathrm{~mm}$ (i.e. with $92 \%$ overlap between windows). For each depth, the power spectra of the backscattered radio-frequency echoes were averaged over 20 acquisitions (corresponding to the 20 acquired B-mode images) to provide $P_{\text {meas }}$. For the complex flow, the data was processed as described for the normal and stop flows, except that 65 and 60 vertical lines were taken on both sides of the valves and echoes were selected with a rectangular window of length $0.4 \mathrm{~mm}$ at 35 depths every $0.031 \mathrm{~mm}$.

A reference measurement was done with fresh porcine blood obtained from a local slaughterhouse. This blood was centrifuged and the plasma and buffy coat were removed, then the RBCs were resuspended in a physiological saline solution to produce a $6 \%$ hematocrit non-aggregating suspension. This reference sample was gently stirred in a beaker. Echoes were windowed as for the studied vein at the same depths and their power spectra were averaged over 20 acquisitions to obtain $P_{r e f}$. This reference power spectrum allows to normalize the average power spectrum $P_{\text {meas }}$ (as described in Eq. (4), section II ).

\section{RESULTS AND DISCUSSION}

For each blood flow condition, quantitative US parametric images of $W, D$ and $\alpha_{0}$ were constructed and superimposed on the gray-scale B-mode images (Figs 2 and 3). The color of each pixel was assigned based on the value of the parameters estimated by the SFSAE. The black pixels in the ROI correspond to rejected solutions of the optimization method (when the estimated packing factor $W$ or diameter $D^{2}$ was found equal to zero, which is unrealistic). The mean values $\bar{W}, \bar{D}$ and $\overline{\alpha_{0}}$ are reported in Table I. For the two structural parameters $\bar{W}$ and $\bar{D}$, statistically significant differences were observed between normal and stopped flow conditions, as well as between blood stagnation and circulation zones in the case of the two closed venous valves; whereas attenuation mean values were quite similar for all cases $(\approx 0.2$ $\mathrm{dB} / \mathrm{cm} / \mathrm{MHz}$ for the normal and stop flows and $\approx 0.3 \mathrm{~dB} / \mathrm{cm} / \mathrm{MHz}$ for the two closed venous valves), as it was expected. In the cases of the normal and stopped flow conditions, quantitative images were 
(a)

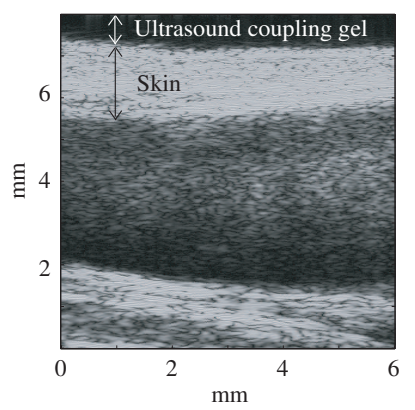

(b)

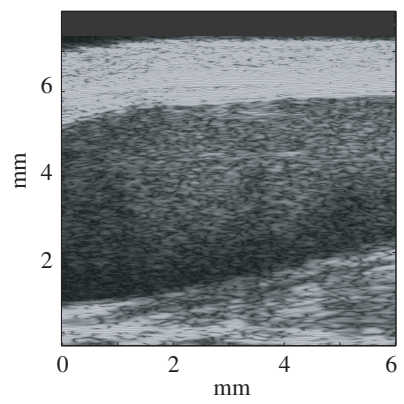

(c)

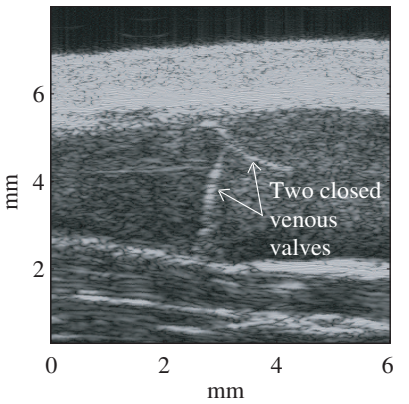

Fig. 1. B-mode images of the three blood flow conditions. (a) Normal blood flow, (b) stop flow with a proximal and distal constrictions and (c) complex flow in the vinicity of two momentarily closed venous valves.

quite different with zones with quasi-disaggregated $\mathrm{RBCs}(1<D<2)$ and zones with bigger aggregates ( $3<D<4$ for the normal flow, and $3<D<6$ for the stopped flow).

In our previous papers, porcine blood sheared in both Couette and tubular flow devices was studied [4], [9]. In these controlled flow experiments, we showed that the SFSAE performed well for $D<7.29$ and for total attenuations between 0.115 and $0.411 \mathrm{~dB} / \mathrm{MHz}$. These bounds define the SFSAE validity domain, i.e. the domain where the SFSAE gives accurate estimates of $W, D$ and $\alpha_{0}$. Outside of these bounds, the SFSAE gave qualitatively satisfactory estimates. In this in vivo study, since the estimates were in the SFSAE validity domain, estimates are expected to be accurate. One may notice that the RBC aggregate size under the in vivo conditions are smaller that those obtained in vitro in previous studies. Indeed, in the current study, the diameters $D$ were between 1.09 and 3.69, whereas $D$ were between 1.60 and 7.29 for the in vitro tube experiment [9]. Nevertheless, the in vitro tube experiment with porcine blood didn't reproduce the in vivo human vein flow condition of this study since the tube diameter and the flow rate were different, and since porcine and human blood have different rheological behaviors.

In figures 2 and 3, one can notice the rejected solutions represented in black pixels. It can be clearly 

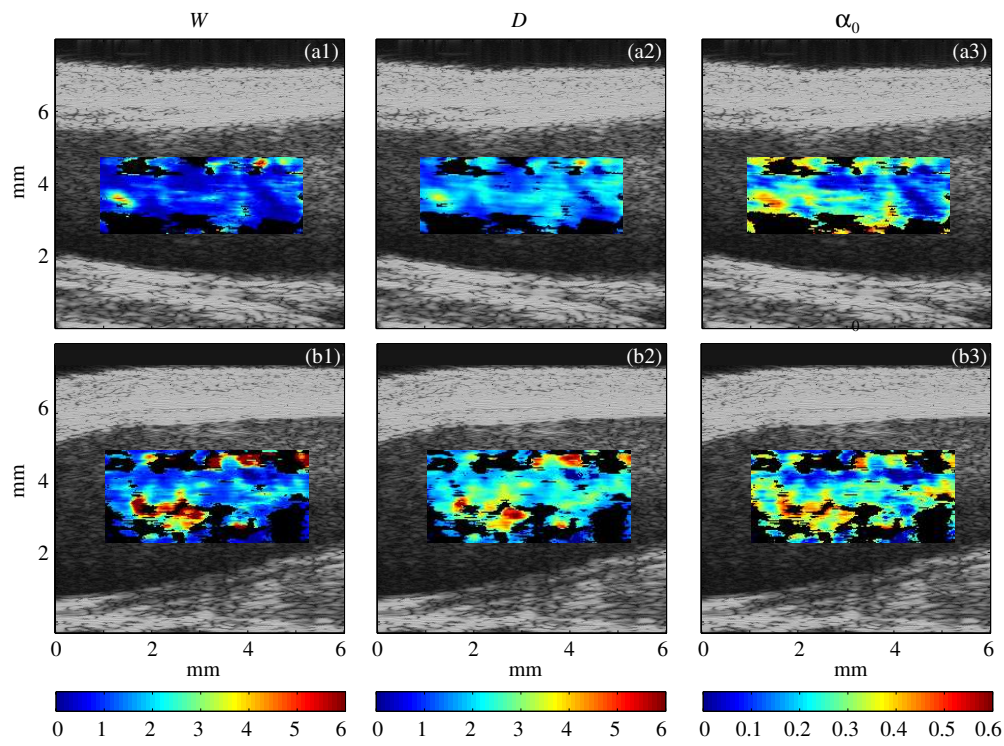

Fig. 2. Quantitative images of blood circulating in the vein superimposed on the gray-scale B-mode images. (a) Normal blood flow, (b) stop flow.
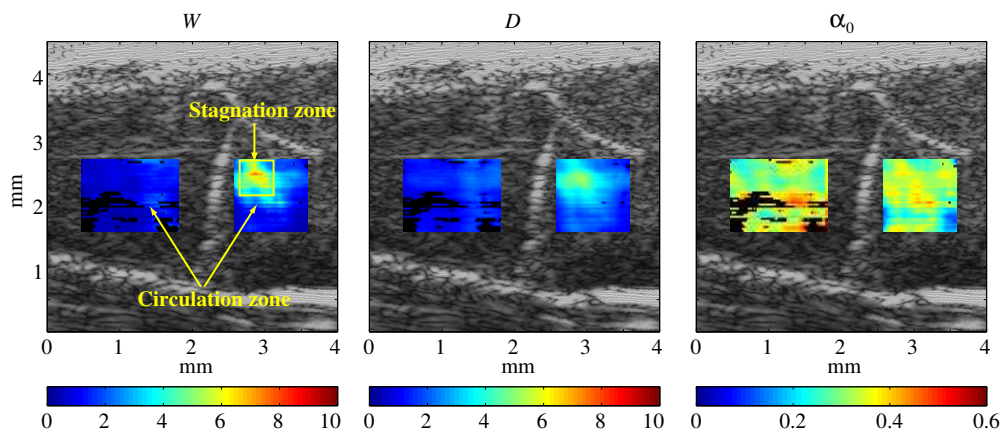

Fig. 3. Quantitative images of blood circulating in the vinicity of the two closed venous valves superimposed on the gray-scale B-mode images.

observed that percentages of rejected solutions were highest for the normal and stop flows $(25 \%$ and $36 \%$, respectively) and for the circulation zone with the two valves (19\%) in comparison with the stagnation zone of the two valves $(0.3 \%)$. In this last case, $D$ and $\alpha_{0}$ were the biggest. It is thus consistent with the observations made in our previous paper [9], where the rejected solutions were more important when the frequency dependence was nearly Rayleigh (small attenuation and quasi-disaggregated RBCs). 
TABLE I

MeAn ESTIMATES of THE PACKING FACTOR $W$, THE AGgREGATE DiAMETER EXPRESSED IN NUMBER OF RBCs $D$ AND THE TOTAL ATTENUATION $\alpha_{0}$ IN DB/CM/MHZ FOR THREE BLOOD FLOW CONDITIONS.

\begin{tabular}{|c|c|c|cc|}
\hline & Normal & Stop & \multicolumn{2}{|c|}{ Complex blood flow } \\
& blood flow & blood flow & Circulation & Stagnation \\
\hline$W$ & $0.91 \pm 0.70$ & $2.17 \pm 1.82$ & $0.86 \pm 0.46$ & $4.20 \pm 1.34$ \\
$D$ & $1.61 \pm 0.59$ & $2.42 \pm 1.13$ & $1.09 \pm 0.66$ & $3.69 \pm 0.81$ \\
$\alpha_{0}$ & $0.221 \pm 0.106$ & $0.246 \pm 0.105$ & $0.342 \pm 0.067$ & $0.318 \pm 0.036$ \\
\hline
\end{tabular}

\section{CONCLUSION}

The SFSAE has been shown to be able of estimating blood backscattering properties in vivo and in situ. Future works should focus on in vivo and in situ assessment of the pathophysiological impact of abnormal RBC aggregation on the cardiovascular system.

\section{ACKNOWLEDGMENTS}

This work was supported by the Canadian Institutes of Health Research (grants $\sharp$ MOP-84358 and CMI-72323), by the Heart and Stroke Foundation of Canada (grant $\sharp$ PG-05-0313), and by the National Institutes of Health of USA (grant $\sharp$ RO1HL078655).

\section{REFERENCES}

[1] F. T. H. Yu, and G. Cloutier, "Experimental ultrasound characterization of red blood cell aggregation using the structure factor size estimator", J. Acoust. Soc. Am., vol. 122, pp. 645-656, 2007.

[2] F. L. Lizzi, M. Astor, T. Liu, C. Deng, D. J. Coleman, and R. H. Silverman, Ultrasonic spectrum analysis for tissue assays and therapy evaluation, Int. J. Imaging Syst. Technol., vol. 8, pp. 3-10, 1997.

[3] T. A. Bigelow, M. L. Oelze, and W. D. OBrien, Estimation of total attenuation and scatterer size from backscatter ultrasound waveforms, J. Acoust. Soc. Am., vol. 117, pp. 1431-1439, 2005.

[4] E. Franceschini, F. T. H. Yu and G. Cloutier, Simultaneous estimation of attenuation and structure parameters of aggregated red blood cells from backscatter measurements, J. Acoust. Soc. Amer., vol. 123, pp. EL85-EL91, 2008.

[5] L. Y. L. Mo and R. S. C. Cobbold, "Theoretical models of ultrasonic scattering in blood", in Ultrasonic Scattering in Biological Tissues, edited by K. K. Shung and G. A. Thieme (CRC, Boca Raton, FL), Chap. 5, pp.125-170, 1993.

[6] V. Twersky, "Low-frequency scattering by correlated distributions of randomly oriented particles", J. Acoust. Soc. Am., vol. 81, pp. 1609-1618, 1987.

[7] K. K. Shung, "On the ultrasound scattering from blood as a function of hematocrit", IEEE Trans. Ultras., Ferroelect., Freq. Contr., vol. SU-26, pp. 327-331, 1982.

[8] S. H. Wang and K. K. Shung, ”An approach for measuring ultrasonic backscattering from biological tissues with focused transducers", IEEE Trans. Biomed. Eng., vol. 44, pp. 549-554, 1997. 
[9] E. Franceschini, F. T. H. Yu, F. Destrempes and G. Cloutier, Ultrasound characterization of red blood cell aggregation with intervening attenuating tissue-mimicking phantoms, submitted. 